\title{
STABILITY AND POST-BUCKLING STATE OF SQUARE COMPOSITE WALLS OF ANISOTROPIC STRUCTURE SPARS UNDER SHEAR
}

\author{
Oleg Mitrofanov ${ }^{1}$, Igors Lebedevs ${ }^{2}$, Vladislavs Turko ${ }^{2}$ \\ ${ }^{1}$ Moscow Aviation Institute (National Research University), Russia; \\ ${ }^{2}$ Riga Technical University, Latvia \\ mitrofanovov@mai.ru,igors.lebedevs@aviatest.lv, vladislav.turko@gmail.com
}

\begin{abstract}
As an object of the research, a square fragment of the wall of the spar of the low- or medium-capacity airplane wing caisson structure is considered. When designing thin spar walls, it is advisable to use anisotropic panels with different values of critical shear stresses depending on the direction of the action of the shear forces in different design cases of loading. The paper assumes that the shape of the panel is close to square, the deflection function is described by trigonometric functions, includes two terms and satisfies the boundary conditions for hinged support. The Bubnov-Galerkin method is used to solve a geometrically nonlinear problem for a square anisotropic panel under shear loading. The presented solution of the geometrically nonlinear problem is reduced to a nonlinear system of two equations with respect to two deflection amplitudes. From the definition of the Erie stress function, analytical expressions for the membrane stresses arising from the loss of panel stability are written down in the paper. When considering a linear problem, an analytical expression for determining the critical flows at the loss of stability of square anisotropic panels is obtained. The above expression for a given tangential flux and stacking allows us to carry out the design calculation and determine the minimum thickness of an anisotropic panel. On the basis of the presented analytical expression, examples of calculations of shear stability of smooth square anisotropic panels are given and the possible effectiveness of anisotropic structures as compared to orthotropic panels is shown when considering two design cases under the action of shear forces that differ in direction and absolute values.
\end{abstract}

Keywords: post-buckling state, stability, anisotropic structure, square panel, shear.

\section{Introduction}

Let us consider a fragment of the wall structure of the wing caisson spar of a low- or mediumcapacity aircraft between the ribs in the root part of the wing, where there are large construction heights, and the wall is designed under the conditions of stability and load-bearing capacity limitations. Let the specified fragment of the wall has a shape close to a square. Note that practically all wing structures of modern passenger airplanes are designed for loads in "straight" cases (for example, case A [1]) with operational overloading nyoper $=2.5$, and in the "reverse" cases (e.g., the case of $D[1]$ ) for overloaded loads nyoper $=-1.0$. That is, the shear force in the "straight" (wing bend up) cases is greater than in the "reverse" (wing bend downwards) cases and it is advisable to use walls of anisotropic structure when designing thin walls of spars. The specified design problem for rectangular long wall fragments from the position of stability is considered in [2]. The monograph [3] notes the practical significance of the analytical solution obtained in [2].

Let us further consider the problems of stability and post-buckling behaviour of an anisotropic square panel under shear in order to further develop techniques for optimal design. In this case, we could assume that the square anisotropic panel should take two design cases under the action of tangential flows of different signs and differing in magnitude, for example, by more than 2 times.

Note that the design problems of rectangular anisotropic walls were considered in [4] under stability and post-buckling state constraints. The methodology for the design of composite panels considering the above constraints is outlined in [5].

The works [6-7] are devoted to the design of load-bearing panels considering the solution of optimal reinforcement problems. The solutions of problems of composite panels stability under shear are given in works [8-9]. Note that analytical solutions of stability and geometrically nonlinear problems can be used when considering the behaviour of defects of the splitting type. The publications [10-11] present the results of computational and experimental works in this direction.

\section{Problem statement and initial relations}

Let us represent the deflection of a square anisotropic panel (with comparable geometric parameters $a \approx b$ ) under the action of tangential flows and the boundary conditions corresponding to the hinged support in the following form 


$$
W=f_{1} \cdot \sin \frac{\pi x}{a} \sin \frac{\pi y}{b}+f_{2} \cdot \sin \frac{2 \pi x}{a} \sin \frac{2 \pi y}{b} .
$$

Let us use the initial relations of composite structures presented in [12]. Let us write down the equation of joint deformation of an anisotropic panel with consideration of geometric nonlinearity

$$
L_{1}(F)-L_{2}(W)=0,
$$

where $F$ - function of Erie stresses.

$$
\begin{gathered}
L_{1}(F)=\frac{1}{E_{y}} \frac{\partial^{4} F}{\partial x^{4}}-g_{31} \frac{\partial^{4} F}{\partial x^{3} \partial y}+g_{22} \frac{\partial^{4} F}{\partial x^{2} \partial y^{2}}-g_{13} \frac{\partial^{4} F}{\partial y^{3} \partial x}+\frac{1}{E_{x}} \frac{\partial^{4} F}{\partial y^{4}}, \\
L_{2}(W)=\left(\frac{\partial^{2} W}{\partial x \partial y}\right)^{2}-\frac{\partial^{2} W}{\partial x^{2}} \frac{\partial^{2} W}{\partial y^{2}} \\
g_{31}=\frac{\eta_{y, x y}+\eta_{x y, y}}{G_{x y}}, g_{22}=\frac{1}{G_{x y}}-\frac{\mu_{x y}}{E_{y}}-\frac{\mu_{y x}}{E_{x}}, g_{13}=\frac{\eta_{x, x y}+\eta_{x y, x}}{G_{x y}}
\end{gathered}
$$

where $E_{x}, E_{y}, G_{x y}, \mu_{x y}$ - averaged characteristics of the multilayer package

$\eta_{y, x y}, \eta_{x y, y}$-influence coefficients of the anisotropic structure [12].

When solving the geometrically nonlinear problem by the Bubnov-Galerkin method, we use the equations

$$
\begin{aligned}
& \int_{0}^{b} \int_{0}^{a}\left[L_{3}(F, W)-L_{4}(W)\right] \sin \frac{\pi x}{a} \sin \frac{\pi y}{b} d x d y=0, \\
& \int_{0}^{b} \int_{0}^{a}\left[L_{3}(F, W)-L_{4}(W)\right] \sin \frac{2 \pi x}{a} \sin \frac{2 \pi y}{b} d x d y=0,
\end{aligned}
$$

where

$$
\begin{gathered}
L_{3}(F, W)=\frac{\partial^{2} F}{\partial y^{2}} \frac{\partial^{2} W}{\partial x^{2}}+\frac{\partial^{2} F}{\partial x^{2}} \frac{\partial^{2} W}{\partial y^{2}}-2 \frac{\partial^{2} F}{\partial x \partial y} \frac{\partial^{2} W}{\partial x \partial y} \\
L_{4}(W)=\frac{1}{\delta}\left[D_{11} \frac{\partial^{4} W}{\partial x^{4}}+2\left(D_{12}+2 D_{33}\right) \frac{\partial^{4} W}{\partial x^{2} \partial y^{2}}+D_{22} \frac{\partial^{4} W}{\partial y^{4}}+4 D_{16} \frac{\partial^{4} W}{\partial x^{3} \partial y}+4 D_{26} \frac{\partial^{4} W}{\partial x \partial y^{3}}\right] .
\end{gathered}
$$

Here and hereafter, $D_{m n}$ are the bending stiffnesses of the anisotropic panel [12].

\section{Applied calculation and design methodology}

Then we substitute the deflection (1) into the joint deformation equation (2). As a result, we obtain the equation

$$
\begin{aligned}
L_{1}(F)= & \left(\frac{\pi^{2}}{a b}\right)^{2}\left\{\frac{f_{1}^{2}}{2}\left[\cos \frac{2 \pi x}{a}+\cos \frac{2 \pi y}{b}\right]+f_{2}^{2}\left[\cos \frac{4 \pi x}{a}+\cos \frac{4 \pi y}{b}\right]+\right. \\
& +4 f_{1} f_{2}\left[\cos \left(\frac{3 \pi x}{a}\right) \cos \left(\frac{\pi y}{b}\right)+\cos \left(\frac{\pi x}{a}\right) \cos \left(\frac{3 \pi y}{b}\right)\right],
\end{aligned}
$$

the solution of which will be a function of the stresses Eri

$$
\begin{gathered}
F=\frac{f_{1}^{2}}{2}\left[A_{01} \cos \frac{2 \pi x}{a}+A_{02} \cos \frac{2 \pi y}{b}\right]+f_{2}^{2}\left[A_{03} \cos \frac{4 \pi x}{a}+A_{04} \cos \frac{4 \pi y}{b}\right]+ \\
+f_{1} f_{2}\left[A_{05} \cos \left(\frac{3 \pi x}{a}\right) \cos \left(\frac{\pi y}{b}\right)+A_{06} \cos \left(\frac{\pi x}{a}\right) \cos \left(\frac{3 \pi y}{b}\right)\right]-\frac{p_{x} y^{2}}{2}-\frac{p_{y} x^{2}}{2}+p_{x y} x y,
\end{gathered}
$$


where $p_{x}, p_{y}, p_{z}-$ normal and tangential stresses applied to the panel.

After considering partial solutions of the homogeneous equation, we can determine the following coefficients, considering the anisotropic structure

$$
\begin{gathered}
A_{01}=A_{03}=\frac{E_{y}}{32} \frac{a^{2}}{b^{2}}, A_{02}=A_{04}=\frac{E_{x}}{32} \frac{b^{2}}{a^{2}}, \\
A_{05}=\frac{4}{a b^{2}} \frac{1}{\frac{1}{E_{y}}\left(\frac{3}{a}\right)^{4}+g_{31}\left(\frac{3}{a}\right)^{3}\left(\frac{1}{b}\right)^{1}+g_{22}\left(\frac{3}{a}\right)^{2}\left(\frac{1}{b}\right)^{2}+g_{13}\left(\frac{3}{a}\right)^{1}\left(\frac{1}{b}\right)^{3}+\frac{1}{E_{x}}\left(\frac{1}{b}\right)^{4}}, \\
A_{06}=\frac{4}{a b^{2}} \frac{1}{\frac{1}{E_{y}}\left(\frac{1}{a}\right)^{4}+g_{31}\left(\frac{1}{a}\right)^{3}\left(\frac{3}{b}\right)^{1}+g_{22}\left(\frac{1}{a}\right)^{2}\left(\frac{3}{b}\right)^{2}+g_{13}\left(\frac{1}{a}\right)^{1}\left(\frac{3}{b}\right)^{3}+\frac{1}{E_{x}}\left(\frac{3}{b}\right)^{4}} .
\end{gathered}
$$

From the definition of the stress function Eri, the stresses in the median surface of the anisotropic plate can be determined

$$
\sigma_{\mathrm{x}}=\frac{1}{\delta} \frac{\partial^{2} \mathrm{~F}}{\partial \mathrm{y}^{2}}, \sigma_{\mathrm{y}}=\frac{1}{\delta} \frac{\partial^{2} \mathrm{~F}}{\partial \mathrm{x}^{2}}, \tau_{\mathrm{xy}}=-\frac{1}{\delta} \frac{\partial^{2} \mathrm{~F}}{\partial \mathrm{x} \partial \mathrm{y}} .
$$

Then, in accordance with the procedure of the Bubnov-Galerkin method, we substitute the deflection (1) and the stress function (5) into equations (3)-(4). After some cumbersome transformations one can obtain a nonlinear system of equations regarding deflection amplitudes under the influence of tangential stresses

$$
\begin{gathered}
\frac{\pi^{4} f_{1}}{4 a^{3} b^{3} \delta}\left[D_{11} b^{4}+2 D_{3} a^{2} b^{2}+D_{22} a^{4}\right]-\frac{256 \pi^{2} f_{2}}{9 a^{2} b^{2} \delta}\left(D_{16} b^{2}+D_{26} a^{2}\right)+ \\
+\frac{\pi^{4}}{2 a b}\left[A_{01} f_{1}^{3}+A_{02} f_{1}^{3}+2 f_{1} f_{2}^{2}\left(A_{05}+A_{06}\right)\right]-\pi^{2}\left[\frac{b f_{1} p_{x}}{4 a}+\frac{a f_{1} p_{y}}{4 b}\right]-\frac{32 f_{2} p_{x y}}{9}=0 \\
\frac{4 \pi^{4} f_{2}}{a^{3} b^{3} \delta}\left[D_{11} a^{4}+2 D_{3} a^{2} b^{2}+D_{22} b^{4}\right]-\frac{64 \pi^{2} f_{1}}{9 a^{2} b^{2} \delta}\left(D_{16} b^{2}+D_{26} a^{2}\right)+ \\
+\frac{\pi^{4}}{a b}\left[8 f_{2}^{3}\left(A_{03}+A_{04}\right)+f_{1}^{2} f_{2}\left(A_{05}+A_{06}\right)\right]-\pi^{2}\left[\frac{b f_{2} p_{x}}{a}+\frac{a f_{2} p_{y}}{b}\right]-\frac{32 f_{1} p_{x y}}{9}=0 .
\end{gathered}
$$

Note that the solution of this system in the general case is only possible numerically.

Now let us consider the problem of shear stability and rewrite the system of equations (8) considering rejection of nonlinear terms with high degrees of deflection amplitude $\left(f_{i} \rightarrow 0\right)$. A simplified system of equations for small deflections in this case is

$$
\begin{aligned}
& \frac{\pi^{4} f_{1}}{4 a^{3} b^{3}}\left[D_{11} b^{4}+2 D_{3} a^{2} b^{2}+D_{22} a^{4}\right]-\frac{256 \pi^{2} f_{2}}{9 a^{2} b^{2}}\left(D_{16} b^{2}+D_{26} a^{2}\right)-\frac{32 f_{2} p_{x y} \delta}{9}=0, \\
& \frac{4 \pi^{4} f_{2}}{a^{3} b^{3}}\left[D_{11} a^{4}+2 D_{3} a^{2} b^{2}+D_{22} b^{4}\right]-\frac{64 \pi^{2} f_{1}}{9 a^{2} b^{2}}\left(D_{16} b^{2}+D_{26} a^{2}\right)-\frac{32 f_{1} p_{x y} \delta}{9}=0 .
\end{aligned}
$$

Then we can write down the determinant of the system of equations (9) with respect to the deflection amplitudes. At $f_{1} \neq 0$ and $f_{2} \neq 0$ we have

$$
\left|\begin{array}{cc}
\frac{\pi^{4} D_{\mathrm{mn}}}{4 a^{3} b^{3}} & -\left(\frac{256 \pi^{2} D_{\alpha \beta}}{9 a^{2} b^{2}}+\frac{32 \mathrm{q}_{x y}}{9}\right) \\
-\left(\frac{64 \pi^{2} D_{\alpha \beta}}{9 a^{2} b^{2}}+\frac{32 \mathrm{q}_{x y}}{9}\right) & \frac{4 \pi^{4} D_{\mathrm{mn}}}{a^{3} b^{3}}
\end{array}\right|=0,
$$


where $\quad D_{\mathrm{mn}}=D_{11} b^{4}+2 D_{3} a^{2} b^{2}+D_{22} a^{4}, D_{\alpha \beta}=D_{16} b^{2}+D_{26} a^{2}$,

from where we can write the quadratic equation with respect to the shear force and find its critical value as a solution of the equation

$$
q_{x y}^{2}+q_{x y} B+C=0
$$

where

$$
B=\frac{10 \pi^{2} D_{\alpha \beta}}{a^{2} b^{2}}, C=\frac{16 \pi^{4} D_{\alpha \beta}^{2}}{a^{4} b^{4}}-\left(\frac{9}{32}\right)^{2} \frac{\pi^{8}}{a^{6} b^{6}} D_{\mathrm{mn}}^{2} .
$$

By entering the designations $D_{m n}=D_{m n} \delta^{3}$ and $D_{\alpha \beta}=D_{\alpha \beta} \delta^{3}$, let us rewrite the solution of equation (11) in the following form

$$
q_{x y}=\frac{\pi^{2} \delta^{3}}{2 b^{2}}\left[-\frac{10 \bar{D}_{\alpha \beta}}{a^{2}}+\sqrt{\left(\frac{10 \bar{D}_{\alpha \beta}}{a^{2}}\right)^{2}-4\left(\frac{16 \bar{D}_{\alpha \beta}^{2}}{a^{4}}-\left(\frac{9}{32}\right)^{2} \frac{\pi^{4}}{a^{6} b^{2}} \bar{D}_{\mathrm{mn}}^{2}\right)}\right] .
$$

Note that when designing load-bearing panels, as a rule, the initial ones are flows, but not stresses. The written equality for a given shear flux allows us to determine the minimum thickness of the panel from the conditions of stability.

Next, we consider the post-buckling behaviour of a thin anisotropic panel under shear and assume that the most likely cause of failure may be the achievement of the limiting tangential stresses $\bar{\tau}_{x y}$. The effective stresses in this case are found from the equation

$$
\tau_{x y}=-\frac{\pi^{2}}{\mathrm{ab} \delta} \Delta_{x y}-p_{x y}
$$

where

$$
\Delta_{x y}=\left[3 A_{03} f_{1} f_{2} \sin \frac{3 \pi x}{a} \sin \frac{\pi y}{b}+3 A_{04} f_{1} f_{2} \sin \frac{\pi x}{a} \sin \frac{3 \pi y}{b}\right] .
$$

Note that after the loss of stability, the anisotropic panel must accept the load, which is determined by the condition of reaching the tangential stresses of $\bar{\tau}_{x y}$. To calculate the ultimate tangential force, let us rewrite equation (13) in the form of equality

$$
\bar{\tau}_{x y} \delta=-\frac{\pi^{2}}{\mathrm{ab}} \Delta_{x y}-p_{x y} \delta=-\frac{\pi^{2}}{\mathrm{ab}} \Delta_{x y}-q_{x y} .
$$

expressing from which the flux $q_{x y}$ can be substituted into the system (8) and obtain two nonlinear equations with respect to the unknown deflection amplitudes $f_{1}$ and $f_{2}$.

Next, to determine the maximum tangential stresses, it is necessary to find the potential-critical points. In this case, considering that the trigonometric function $\Delta_{x y}$ has a periodic character, it is reasonable to choose a potential-critical point with coordinates.

Let us rewrite the system (8) in the form

$$
\begin{gathered}
\frac{\pi^{4}}{4 a^{3} b^{3}} \delta^{3}\left[\bar{D}_{11} b^{4}+2 \bar{D}_{3} a^{2} b^{2}+\bar{D}_{22} a^{4}\right]-\frac{256 \pi^{2} f_{2}}{9 a^{2} b^{2} f_{1}} \delta^{3}\left(\bar{D}_{16} b^{2}+\bar{D}_{26} a^{2}\right)+ \\
+\frac{\pi^{4} \delta}{2 a b}\left[A_{01} f_{1}^{2}+A_{02} f_{1}^{2}+2 f_{2}^{2}\left(A_{05}+A_{06}\right)\right]=\frac{32 f_{2} q_{x y}}{9 f_{1}}, \\
\frac{4 \pi^{4} \delta^{3}}{a^{3} b^{3}}\left[\bar{D}_{11} a^{4}+2 \bar{D}_{3} a^{2} b^{2}+\bar{D}_{22} b^{4}\right]-\frac{64 \pi^{2} f_{1} \delta^{3}}{9 a^{2} b^{2} f_{2}}\left(\bar{D}_{16} b^{2}+\bar{D}_{26} a^{2}\right)+ \\
+\frac{\pi^{4} \delta}{a b}\left[8 f_{2}^{2}\left(A_{03}+A_{04}\right)+f_{1}^{2}\left(A_{05}+A_{06}\right)\right]=\frac{32 f_{1} q_{x y}}{9 f_{2}},
\end{gathered}
$$


where the designations $D_{m n}=D_{m n} \delta^{3}$.

Then, from expression (15), considering (14), we can express the effective shear flux $q_{x y}$ and substitute it in the system (16). As a result, we obtain a system of nonlinear equations with respect to the two unknown deflection amplitudes to determine the stress-strain state in the post-buckling state for an anisotropic panel at a given thickness $\delta$. Note that such a system can be solved numerically using software packages such as Mathcad.

\section{Calculation of anisotropic square panels stability}

Let us consider, for example, the calculation of stability of a square panel with total thickness $\delta=1 \mathrm{~mm}$ made of carbon fiber reinforced plastic type KMU-4 with geometric dimensions $a^{*} b=100^{*} 100 \mathrm{~mm}$. Table 1 shows the critical forces determined by formula (12). The presented values illustrate the possibility of using panels with anisotropic structure. The last column of the table shows the critical force ratios for square panels in anisotropic (given in lines 1-2 and 4-5) and orthotropic layups (given in lines 3 and 6).

Critical shear forces of anisotropic square panels

Table 1

\begin{tabular}{|c|c|c|}
\hline Laying & $\boldsymbol{q}_{\boldsymbol{x} y}, \mathbf{k g f} \cdot \mathbf{m m}^{2}$ & $\begin{array}{c}\text { Comparative } \\
\text { coefficients }\end{array}$ \\
\hline $45^{\circ} / 45^{\circ} / 0^{\circ} / 0^{\circ}$ & 3.62 & 0.74 \\
\hline$-45^{\circ} /-45^{\circ} / 0^{\circ} / 0^{\circ}$ & 6.33 & 1.29 \\
\hline $45^{\circ} / 0^{\circ} / 0^{\circ} /-45^{\circ}$ & 4.91 & 1.0 \\
\hline $30^{\circ} / 30^{\circ} / 0^{\circ} / 0^{\circ}$ & 3.48 & 0.76 \\
\hline$-30^{\circ} /-30^{\circ} / 0^{\circ} / 0^{\circ}$ & 5.83 & 1.27 \\
\hline $30^{\circ} / 0^{\circ} / 0^{\circ} /-30^{\circ}$ & 4.6 & 1.0 \\
\hline
\end{tabular}

Let us return to the design problem mentioned at the beginning of this paper for the stability conditions of an anisotropic wall, which can be loaded with two design cases with different directions and values of the cutting forces. Considering the results given in Table 1, it is possible to use similar layouts to select rational wall thicknesses from the stability conditions for different cases. Note that an orthotropic structure may not be optimal compared to an anisotropic wall for the maximum load case. In the practical implementation of an anisotropic structure from the structural and technological constraints will also require the addition of layers to the wall laying, for example, with an angle of reinforcement $\varphi=90^{\circ}$. In the example considered (Table.1) for a wall made of carbon fiber, the values of critical shear flows, depending on the direction of the reinforcement angles, differ by $1.67 \ldots .1 .74$ times.

\section{Conclusions}

This paper presents an analytical solution of the geometrically nonlinear problem for a square anisotropic panel under the action of shear forces, which is reduced to the solution of a nonlinear system of two equations. An analytical expression for determining critical flows at loss of stability is obtained in explicit form. The presented solutions can be used to calculate square structurally anisotropic panels under shear. Based on the obtained solution, a method for determining the optimal thicknesses of square anisotropic panels under shear under stability constraints is proposed.

\section{References}

[1] Avdonin A.S., Figurovsky V.I. Calculation of Strength of Aircraft. Moscow-Mashinostroenie, 1985. 440p.

[2] Balabukh L.I. Stability of plywood plates // Technics of Air Fleet. 1937. No. 9. pp. 19-38.

[3] Falzon B. G., Aliabadi M. Buckling and Postbuckling Structures: Experimental, Analytical and Numerical Studies. 10.1142, 2008, 506 p.

[4] Mitrofanov O.V. Designing thin walls of spars of anisotropic structure by the post-buckling state for the wing of a light aircraft // Natural and Technical Sciences, 2013, No. 6, pp.256-260. 
[5] Mitrofanov O. Post-Buckling State Estimation and Load-Bearing Composite Panels Design for Compression and Shear. AIP Conference Proceedings 2125, 030059 (2019); DOI: $10.1063 / 1.5117441$

[6] Mikhailovsky K.V., Baranovski S.V. Methodology of wing design of polymer composite materials on the basis of parametric modeling. Part 3. The Choice and Substantiation of Optimal Schemes of Reinforcing Strength Elements. Izvestia of Higher Educational Institutions. Mechanical engineering. 2018. No. 5 (698). C. 75-84. DOI: 10.18698/0536-1044-2018-5-75-84.

[7] Grishin V.I., Dzyuba A.S., Dudarkov Y.I. Strength and stability of elements and joints of aircraft structures from composites. -Moscow Fizmatlit. 2013. 270p.

[8] Lopatin A., Korbut Y.. Buckling of clamped orthotropic plate in shear. Composite Structures COMPOS STRUCT. 76. 2006, pp. 94-98. DOI: 10.1016/j.compstruct.2006.06.014.

[9] Lopatin A., Morozov E., Shatov A. Buckling of the composite anisogrid lattice plate with clamped edges under shear load. Composite Structures. 159, 2016. DOI: 10.1016/j.compstruct.2016.09.025.

[10] Chermoshentseva A. S., Pokrovskiy A. M., Bokhoeva L.A. The behavior of delaminations in composite materials - experimental results. IOP Conf. Series: Materials Science and Engineering 116 (2016) 012005 DOI:10.1088/1757-899X/116/1/012005.

[11] Bokhoeva L. A., Bochektueva E. B. An experimental research of the laminated composite plates stability. IOP Conference Series: Materials Science and Engineering. III International Conference of Young Scientists on Contemporary Problems of Materials and Constructions. 2019. C. 012027. DOI:10.1088/1757-899X/684/1/012027.

[12] Vasiliev V. V., Morozov E. V. Advanced Mechanics of Composite Materials and Structures. Amsterdam: Elsevier, 2018, 900 p. 